\title{
The rise of quantum materials
}

\author{
Emergent phenomena are common in condensed matter. Their study now extends beyond strongly \\ correlated electron systems, giving rise to the broader concept of quantum materials.
}

Measured in terms of publication output, condensed-matter physics is the largest field in physics ${ }^{1}$. Its size is a consequence of its breadth: the study of systems in their 'condensed' phases can be applied to an almost limitless range of problems such as magnetism, superconductivity and superfluidity, to name three examples out of many. A cornerstone of this approach is the concept of symmetry breaking, the idea that a condensed phase has a lower symmetry than its uncondensed counterpart (for instance, a solid has a lower symmetry than a gas). The framework through which these phenomena were first understood is the Landau-Ginzburg theory of phase transitions: by identifying a suitable order parameter that reflects the underlying symmetry of the system (such as a material's density, to continue with our solid-state example), it is possible to pinpoint the conditions that are required for that symmetry to become manifest.

The overarching theme for condensedmatter physics in the 1960s and 1970s was therefore a continual search for order. Armed with beautiful ideas rooted in symmetry - concepts that pervade physics well beyond condensed matter, in fact - physicists focused on identifying the relevant and, crucially, measurable order parameters that pertained to their system of choice. Inevitably, work tended to concentrate on the hardest problems to crack - typically those in which there was no discernible order parameter or, if there was, that were characterized by an unusual degree of complexity.

As these problems persisted into the 1980s, two crucial developments occurred. Firstly, the realization that there were exceptions to the Landau-Ginzburg paradigm, such as the topological order that underlies the fractional quantum Hall effect. Secondly, the discovery of hightemperature superconductivity brought about a surge of interest in so-called strongly correlated electron systems. Of course, the many-body problem had been known since the days of Nevill Mott, but a plethora of problems linked more or less directly to superconductivity, such as heavy fermions, quantum criticality and the physics of the pseudogap, combined with the rapid growth of the computational power scientists had access to, brought about a fresh urgency to attack the issue head-on.

Although the mechanism of superconductivity continues to be elusive, the era of strongly correlated electron systems has brought about a number of significant developments. Perhaps the most important of these isn't strictly a scientific discovery, however. Rather, it has been a change in perspective. The study of non-trivial or 'exotic' electronic properties of materials has fostered an appreciation for a variety of emergent phenomena resulting from strong correlation effects - cooperative behaviours that cannot be predicted from the properties of individual electrons. These include emergent excitations such as monopoles ${ }^{2}$ and skyrmions ${ }^{3}$, the description of which is, in many ways, simpler than the original 'vacuum' of excitations from which they arise.

This taste for emergent properties coincided with the experimental discovery of topological insulators, which was made on the back of large strides forward in the understanding of electronic states in terms of topological invariants essentially a geometric property of the electron wavefunction ${ }^{4}$. Although part of a wider surge in interest in topologically protected states of matter, the excitement surrounding topological insulators was principally due to the robust nature of the metallic surface states that characterized them, and the relatively loose requirements for their realization: neither strong electron-electron interactions nor lowdimensionality are required. Moreover, as if to make the point even stronger, these discoveries came almost simultaneously with a flurry of spectacular observations in graphene, including the observation of the fractional quantum Hall effect ${ }^{5,6}$. This is a system made up of $s p^{2}$ electrons - hardly the definition of a strongly correlated material - and yet it displays the hallmarks of topological order, which include dissipationless transport and emergent particles with fractional charge and statistics.
As it has become clear that the study of emergent properties is no longer restricted to strongly correlated electron systems, a new, broader description has become necessary. And the term that seems to be gaining currency on departmental websites and research programmes is quantum materials. Indeed, conferences on the topic have started to spring up - for example, the Big Ideas in Quantum Materials workshop held in La Jolla, California, last December.

Of course, on a trivial level all materials exist thanks to the laws of quantum mechanics, and there are cynics who will privately wonder if the description isn't too broad and, well, catchy for its own good. But given the history of condensed-matter physics that we have just outlined, there are good reasons to embrace quantum materials. In essence, they provide a common thread linking disparate communities of researchers working on a variety of problems at the frontiers of physics, materials science and engineering.

At face value, these problems are quite distinct: think of the recent success of groups hunting for emergent particles in solids (last year's flurry of results on Weyl fermions ${ }^{7}$ are a case in point), and the challenges faced by others looking to harness the promising optoelectronic properties of van der Waals materials for devices and applications. A little further afield, consider the strides forward being made in developing platforms for achieving quantum computers and, by dropping the semantic distinction between material and matter, the control afforded by designer quantum matter platforms synthesized from cold atoms, ions, defects in solids, circuit-QED architectures, and solid-state heterostructures. All these communities face formidable challenges and opportunities. And they all just know they are working on quantum materials.

\footnotetext{
References

1. Sinatra, R. et al. Nature Phys. 11, 791-796 (2015).

2. Castelnovo, C., Moessner, R. \& Sondhi, S. L. Nature 451, 42-45 (2008)

3. Mühlbauer, S. et al. Science 323, 915-919 (2009).

4. Moore, J. E. Nature 464, 194-198 (2010).

5. Du, X. Nature 462, 192-195 (2009).

6. Bolotin, K. I. et al. Nature 462, 196-199 (2009)

7. Nature Phys. 11, 697 (2015).
} 